Voix et Images

voixetimages

\title{
Enfance, famille, deuil
}

Pascal Riendeau

Volume 39, numéro 3 (117), printemps-été 2014

URI : https://id.erudit.org/iderudit/1026218ar

DOI : https://doi.org/10.7202/1026218ar

Aller au sommaire du numéro

Éditeur(s)

Université du Québec à Montréal

\section{ISSN}

0318-9201 (imprimé)

1705-933X (numérique)

Découvrir la revue

Citer ce compte rendu

Riendeau, P. (2014). Compte rendu de [Enfance, famille, deuil]. Voix et Images, 39(3), 118-123. https://doi.org/10.7202/1026218ar d'utilisation que vous pouvez consulter en ligne.

https://apropos.erudit.org/fr/usagers/politique-dutilisation/ 


\author{
R O M A N \\ Enfance, famille, deuil \\ $+++$
}

PASCAL RIENDEAU

Université de Toronto

Lauréat du prix Robert-Cliche en 2007 pour son premier roman, Balade en train assis sur les genoux du dictateur ${ }^{1}$, Stéphane Achille en signe un second, Corbeau et Novembre ${ }^{2}$, dans lequel l'enfance joue un rôle primordial. Dès le départ, on constate un léger déplacement: les noms du titre ne sont pas ceux des protagonistes, mais plutôt ceux de personnages secondaires. La place marginale de ces derniers dans le récit va de pair avec la marginalité de leur mode de vie simple, eux qui vivent dans un chalet construit de manière artisanale, sans électricité, situé en dehors du village, sur un terrain qui ne leur appartient pas. Ces personnages qui, dans les années 1980, continuent de mener une vie communautaire demeurent une source de distraction pour les résidents du village où habitait le narrateur du roman, Charles-Alexandre Dulong. La structure de ce roman de facture classique est divisée en deux parties présentées en alternance. Dans la première, en 2009, le narrateur apprend la mort de sa mère et ne sait pas trop comment vivre son deuil autrement qu'en se rendant au travail, comme si de rien n'était: «Je n'avais pas pensé en me pointant ici ce matin que ce n'était pas si simple, être en deuil. Il faut marcher au pas et bien faire l'endeuillé. Ça peut vite devenir compliqué. » (13) Dans la seconde, il raconte comment, un quart de siècle plus tôt, des événements multiples ont irrémédiablement changé sa vie, alors qu'il n'avait que dix ans, dans ce village dont on ne connaîtra jamais le nom. Le chalet que Corbeau et Novembre ont construit et occupé jusqu'à l'été 1984 représente pour le protagoniste le symbole de son passé trouble, de son malheur. Le récit d'enfance nous fait comprendre, en quelque sorte, comment Dulong est devenu ce personnage obsédé et maniaque. Avocat de formation, il supervise la rédaction de guides d'utilisation d'une entreprise d'électronique. Ses préoccupations pour les détails frôlent souvent le ridicule, ce qui permet à l'ironie de s'infiltrer dans le roman au moment opportun.

Les jours presque identiques de l'été 1984 ont d'abord pris une tournure différente par l'arrivée inattendue de Kevin, un garçon qui a presque le même âge que CharlesAlexandre. Après avoir quitté une secte dirigée par un mystérieux patriarche, ne pouvant

$$
+++
$$

1 Stéphane Achille, Balade en train assis sur les genoux du dictateur, Montréal, VLB éditeur, 2007, 186 p.

2 Stéphane Achille, Corbeau et Novembre, Montréal, XYZ éditeur, 2013, 435 p. 
pas trouver d'endroit où se loger à leur arrivée au village, Kevin et Jocelyne, la femme qui l'accompagne - est-ce sa mère ou non? - , s'installent chez les Dulong. Ni Kevin ni Charles-Alexandre ne connaissent leur père respectif. Suzanne, la mère autoritaire de celui-ci, directrice de l'école du village, a toujours refusé de lui révéler son identité. Ces secrets constituent des éléments importants du suspense et ils contribuent à entretenir l'étrange relation entre le fils et sa mère, qui durera jusqu'à ce que celle-ci meure. Malgré la lourdeur qui parcourt le roman, on assiste à quelques moments humoristiques grâce à Kevin. Jusqu'à son arrivée au village, le garçon a vécu à l'écart du monde, sans fréquenter l'école. Il ne sait pas ce qu'est le Canada, à quoi sert une banque, pourquoi les enfants vont à l'école, et il ignore comment interpréter ce qu'il voit à la télévision. Cela crée un déséquilibre dans le nouvel univers qui l'entoure: au milieu de l'été, «Kevin fai[t] encore mal la différence entre les films à Ciné-Quiz et la réalité» (141). On assiste ensuite à la dispersion du petit groupe d'amis de Charles-Alexandre au sein duquel la moquerie et la mesquinerie dominent, mais c'est la disparition de l'un d'entre eux, Jean-François, surnommé Botte-d'eau, qui transforme cet été en véritable drame. Achille maintient la tension en multipliant la présence de personnages susceptibles d'avoir enlevé le garçon. Ce qui caractérise le récit de 1984, c'est qu'il est écrit du point de vue de l'enfant, mais avec un regard rétrospectif ambivalent. Nous avons simultanément le point de vue de l'enfant, mais jumelé à une réflexion d'adulte. Le choix d'une langue normative, voire soutenue pour les dialogues participe également à cette ambivalence.

Le récit au présent se concentre sur une période de quelques jours, de la mort de la mère du narrateur jusqu'à sa crémation. L'ironie présente dans le roman se manifeste surtout à travers les commentaires que fait le protagoniste sur ses méthodes de travail et sur sa façon de vivre. Solitaire, misanthrope, possédant une passion presque enfantine pour les animaux et surtout les émissions de télévision portant sur les animaux, Dulong habite un appartement dans un immeuble situé au milieu d'un quartier industriel où il est presque certain de ne croiser personne. Il va jusqu'au bout de son obsession, et c'est d'ailleurs ce qui justifie toute la tension dramatique, car la trame narrative du présent, centrée sur une petite erreur dans un courriel que Dulong a envoyé à un autre employé, reste bien mince. Le projet qu'il envisage pour récupérer un courriel qu'il imagine incriminant relève presque du délire. Corbeau et Novembre, c'est aussi le long chemin introspectif d'un personnage qui, à trente-cinq ans, cherche à mieux comprendre qui il est: «Faire fonctionner ma mauvaise foi est une capacité que j'ai développée il y a longtemps, mais le faire en toute conscience est un nouveau jeu pour moi. Enfin, j'ai trouvé un jeu pour lequel je semble posséder un talent naturel.» (420) Le roman de Stéphane Achille n'est pas sans longueurs, et on se plaît à imaginer ce même personnage obsédé dans un univers troublé, mais repris dans un texte plus concis.

Les fausses couches ${ }^{3}$ de Steph Rivard nous entraîne dans un tout autre univers, celui d'une famille désaxée qui habite une grande maison à la campagne, quelque part en

$$
+++
$$

3 Steph Rivard, Les fausses couches, Montréal, Les éditions de ta mère, 2013, 143 p. 
Mauricie. Ce premier roman de l'auteur donne la parole à Will, un garçon de douze ans qui raconte une période de sa vie avec sa famille élargie. Les onze membres du clan ne quittent pratiquement jamais la maison, qui donne parfois l'impression d'être vivante elle aussi. Rivard a réussi à aller bien au-delà du simple récit d'enfance tout en trouvant un ton et un style uniques. Il n'est pas impossible de voir dans le récit des traces d'Émile Ajar, de Réjean Ducharme ou de Gaétan Soucy, auteurs qui ont réussi à donner une voix forte et originale à des narrateurs enfants ou adolescents. Mais toute filiation directe reste bien arbitraire. La parole de Will, si elle semble emprunter à d'autres jeunes narrateurs célèbres, n'est pas qu'une simple imitation. D'ailleurs, la singularité de son énonciation relève de sa nature variable. Le narrateur nous prévient indirectement de cette possible incongruité dans un préambule intitulé «Staccato »: «Dans ma tour, j'invente les mots que je pourrai dire et les choix que je pourrai faire un jour. J'y suis l'adulte que je deviendrai une fois sorti de ma chrysalide qui sent le cercueil. Je m'accouche.» (12) Il faut y lire la proposition d'un pacte avec le lecteur qui stipule que l'énonciation oscillera entre une expression enfantine et une autre plus savante. Aux pensées étranges ou naïves d'un garçon de douze ans qui vit au milieu d'une famille déjantée se superposent celles d'un auteur habile avec les mots qui offre un regard ironique et parfois cruel sur la famille ou sur lui-même. Cette oscillation énonciative habilement tissée permet au lecteur de mieux accepter ce qui pourrait autrement ressembler à une contradiction stylistique.

La longue saison que raconte Will peut s'apparenter à une catastrophe familiale : la grand-mère meurt, le père les abandonne tous, l'oncle Édouard se retrouve dans une "grande maison" qu'on imagine être un hôpital psychiatrique et la tante Bernadette est emportée par une ambulance après une crise de folie sans que l'on sache si elle réussira à s'en sortir. Juste avant le départ définitif du père, au moment où ses parents vont poursuivre leur querelle à l'extérieur, durant la nuit, une prémonition s'empare de Will: «Dehors, ça ressemblait à la fin d'une époque. Les années n'avaient plus de place pour s'imprimer dans le temps, dans ma chair, ou dans la pluie qui attendait le moment idéal pour se mettre à pleurer.» (47) Parmi une série d'événements inhabituels, son cousin Nick et sa sœur Lucille forcent Will à tuer violemment sa vieille chatte, Mindy. Quant à sa tante Bernadette, elle se sait malade et prévient Will qu'il risque d'«attraper la même maladie qu'elle» (65). Elle le met également en garde sur ce qui risque de perpétuer les problèmes de la lignée: «- Ton cousin est encore en train d'se vider dans ta sœur, l'écœurant. C'est pas en continuant d'la fourrer comme un pain d'même qu'y va nous aider à stopper l'hémorragie d'avortons. » (66)

La mort de sa grand-mère provoque chez le garçon une transformation que l'on constate dans sa façon de s'interroger sur son identité: "Chaque matin, je suis ici, chaque matin, je me revois et je tente de me convaincre que ça ira mieux bientôt, je repense à hier, je redeviens la partie de moi que je n'ai pas été, j'essaie de moins m'enliser [...]. Je veux reprendre ce qui m'appartient [...], je joue au plus fort, et je m'écroule en réalisant que ça ne donne rien. Je ne sais pas vers où je marche.» (101) Sa réflexion sur le deuil devient une prise de conscience de la place qu'il occupe dans le monde, au-delà du cercle familial. Ce qui caractérise l'écriture des Fausses couches, c'est qu'un tel passage est immédiatement suivi d'un autre plus léger, mais surtout 
plus caractéristique des pensées d'un garçon comme Will. Ce va-et-vient entre la gravité et la légèreté, entre une langue soutenue et une langue plus populaire ou vulgaire, bref tous ces changements de registres permettent à Rivard d'éviter le piège d'une narration convenue ou caricaturale. Will nous surprend presque toujours, soit par les événements inattendus ou étranges qu'il raconte, soit par le renversement provoqué par un autre discours.

Après avoir publié quelques recueils de poèmes, Marjolaine Deschênes propose Fleurs au fusil ${ }^{4}$, son premier roman, mais dont la version initiale aurait été rédigée à l'automne 2001. Divisé en trois parties principales et un long épilogue, le roman se rapproche souvent de l'essai, présent à travers les nombreuses pensées de la narratrice, Viviane Videloup, elle-même romancière et philosophe. Fleurs au fusil est truffé de références multiples, surtout à la littérature romantique et à la philosophie. La première partie s'ouvre d'ailleurs sur une section intitulée «Les décombres du romantisme ", où il est question de l'enfance de la narratrice et d'une étonnante association entre les activités de son père et les auteurs romantiques: «Je suis née dans les décombres du romantisme, car c'est bien à l'orée du dix-neuvième siècle que les hommes se sont mis en tête de bourrer les animaux de paille [...]. Mon père était le dernier de ces drôles, à rembourrer tout ce qui ne respire plus de matières douteuses afin d'en tirer une apparence de vie. J'espérais qu'il soit le dernier. » (15) L'établissement d'un parallèle entre Novalis, dont une citation est placée en épigraphe, et un père taxidermiste et violent peut surprendre. Cependant, Deschênes réussit à créer des liens inattendus qui donnent une couleur singulière à son texte.

Résolue à ne plus écrire, surtout pas de roman, la narratrice décide de prendre un congé sabbatique après la mort de Louis, son voisin et son père substitut, celui qu'elle appelle son « repère». Elle se rend en Belgique pour retrouver son ami Laurent Louve. À Bruxelles, elle découvre un café - fréquenté à une époque par René Magritte ainsi que par de nombreux peintres et poètes surréalistes - dont les murs sont couverts d'aphorismes. Bien que son nom ne soit pas mentionné, un des auteurs qui a dû fréquenter l'endroit et qui y a assurément laissé une trace écrite est Louis Scutenaire. L'un de ses aphorismes rappelle justement la situation de la narratrice : «Il n'a pas une grande facilité pour écrire, il a une grande difficulté à ne pas écrire ${ }^{5}$.» Non seulement Viviane semble incapable de tenir sa promesse de ne plus écrire, mais ses pensées et ses souvenirs la ramènent presque toujours à l'écriture, celle d'avant, qu'elle explique et contextualise, et celle du nouveau projet qu'elle a entamé. Deschênes s'est même permis de recycler les titres de ses ouvrages antérieurs et de les présenter comme s'ils avaient été publiés par la protagoniste. Ainsi, les livres de poésie de l'auteure deviennent des romans de la narratrice. Ce recyclage devient d'autant plus judicieux que Rodenbach ou l'amertume, roman publié par la narratrice, mais

$$
+++
$$

4 Marjolaine Deschênes, Fleurs au fusil, Chicoutimi, La peuplade, 2013, 174 p. 5 Louis Scutenaire, Mes inscriptions. 1943-1944, Paris, Allia, 2007, p. 178. 
qui n'existe pas, est en réalité le titre initial du manuscrit de Fleurs au fusil ${ }^{6}$. «Rodenbach ou l'amertume» est repris comme titre de la dernière section de la première partie, dans laquelle on peut lire un résumé de l'ouvrage qui explique (indirectement) l'échec du projet romanesque non de la narratrice, mais de l'auteure: «Bien sûr ça n'a pas marché. Un roman est en soi romantique. Voilà pourquoi je n'en écrirais plus.» (44)

Roman familial, récit de soi, Fleurs au fusil se transforme en une histoire d'amitié et d'amour non romantique. La narratrice cherche bien à marquer la différence entre le sens commun et le sens littéraire et philosophique du romantisme. Elle adresse d'ailleurs un reproche à son ami belge à ce sujet: "Comme la plupart des romantos de ce monde, Laurent mélangeait tout. Mes idées n'avaient rien à voir avec le romantisme insipide associé aux relations amoureuses. » (50-51) Bien que la distinction de la narratrice s'avère juste, le roman glisse parfois d'un sens à l'autre. Dans ce roman où les références implicites aux fleurs littéraires abondent, la recherche de la fleur bleue de Novalis - l'auteur le plus cité - n'est jamais mentionnée. En revanche, certaines scènes (notamment les retrouvailles de Viviane et Fleure, une amie d'enfance avec qui elle aura une aventure sexuelle) relèvent plus du sentimentalisme, c'est-à-dire que l'on pourrait les qualifier de «fleur bleue», selon le sens dérivé. Outre Novalis, Jean-Jacques Rousseau, Georges Rodenbach, Paul Ricœur et Nancy Huston sont placés au centre d'une mosaïque d'auteurs que convoque la narratrice afin d'explorer ses pensées et son écriture. Comme elle affirme avoir été lauréate du prestigieux prix Raymond-Klibansky, on aurait pu croire que Viviane Videloup exprimerait une vision philosophique plus complexe. On peut se demander pourquoi Deschênes n'a pas donné à sa narratrice une voix d'intellectuelle plus convaincante. Ce roman, qui laisse une place importante à la correspondance à l'ancienne, se termine précisément par un échange épistolaire entre Viviane et Fleure. Devant la longue missive de Viviane, on reste parfois surpris de la teneur des propos à l'égard de Huston et de Ricœur : «Je l'aime, même si la mode et l'idéologie féministes la méprisent. [...] Elle est venue tard Huston, mais pas trop. [...] Cette femme a mis le doigt sur mon sexe.» (156) Ailleurs, elle fait preuve d'une aimable bienveillance : «À Paul il manque parfois le mot de trop: le mot "femme". À Nancy il manque parfois la lenteur et la prudence d'une pensée qui se voudrait juste plutôt que cinglante. » (157) Il s'agit là d'une critique pertinente, et on aurait souhaité en lire davantage.

Ces trois romans qui, à des degrés divers et de manières fort différentes, proposent un récit d'enfance ou une histoire familiale, posent aussi un regard pertinent sur le deuil. Achille insiste à la fois sur l'introspection et la distance, Rivard sur l'onirisme

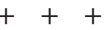

6 C'est ce que Deschênes a expliqué au site Web littéraire La recrue du mois: «Le manuscrit a porté plusieurs titres provisoires, dont La cabane rose et Rodenbach ou l'amertume. J'ai choisi le titre Fleurs au fusil très tard, juste avant l'impression.» En ligne: http://larecrue.net/2014/01/marjolaine-deschenes-questionnaire/(page consultée le 9 mai 2014). 
et l'humour noir, tandis que Deschênes mise sur l'ironie et le lyrisme. Parmi les hasards que réserve une telle chronique, il y a les personnes à qui les auteurs dédient leur livre. Rivard présente deux dédicaces : l'une à un petit groupe de personnes et l'autre à Catherine Mavrikakis. Marjolaine Deschênes, elle, offre son livre à la santé de quelques auteurs, dont Catherine Mavrikakis. Ce rapprochement qu'ils établissent avec l'auteure de Fleurs de crachat $^{7}$ montre que celle-ci est une écrivaine qui s'impose de plus en plus dans le paysage littéraire québécois. On en voit bien des traces dans des œuvres variées.

$$
++
$$

7 Catherine Mavrikakis, Fleurs de crachat, Montréal, Leméac, 2005, 198 p. 